\title{
GAN-aided Serial Dependence Study in Medical Image Perception
}

\author{
Zhihang Ren \\ peter.zhren@berkeley.edu \\ University of California, Berkeley \\ Berkeley, California, USA
}

\begin{abstract}
Medical imaging has been critically important for the health and well-being of millions of patients. Although deep learning has been widely studied in medical imaging area and the performance of deep learning has exceeded human's performance in certain medical diagnostic tasks, detecting and diagnosing lesions still depends on the visual system of human observers (radiologists), who completed years of training to scrutinize anomalies. Routinely, radiologists sequentially read batches of medical images one after the other. A basic underlying assumption of radiologists' precise diagnosis is that their perceptions and decisions on a current medical image are completely independent from the previous reading history of medical images. However, recent research proposed that the human visual system has visual serial dependencies (VSDs) at many levels. VSD means that what was seen in the past influences (and captures) what is seen and reported at this moment. Our pilot data via naive artificial stimuli has shown that VSD has a disruptive effect in radiologic searches that impairs accurate detection and recognition of tumors or other structures. However, the naive artificial stimuli have been noted by both untrained observers and expert radiologists to be less authentic. In this project, we will generate authentic medical images via Generative Adversarial Networks (GANs) in order to replace the simple stimuli in future experiments. The rationale for the proposed research project is that once it is known how serial dependence arises and how it impacts visual search, we can understand how to control for it. Hence, the accuracy of diagnosis via medical imaging can significantly improve. The specific goals of this project are to establish, identify and mitigate the impact of VSD on visual search tasks in clinical settings.
\end{abstract}

\section{CCS CONCEPTS}

- Computing methodologies $\rightarrow$ Computer vision; Cognitive science.

\section{KEYWORDS}

serial dependence; medical image perception; generative adversarial networks

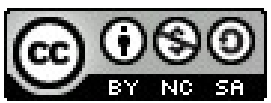

This work is licensed under a Creative Commons Attribution-NonCommercialShareAlike International 4.0 License.

MM '21, October 20-24, 2021, Virtual Event, China.

(C) 2021 Copyright held by the owner/author(s).

ACM ISBN 978-1-4503-8651-7/21/10.

https://doi.org/10.1145/3474085.3481028
ACM Reference Format:

Zhihang Ren. 2021. GAN-aided Serial Dependence Study in Medical Image Perception. In Proceedings of the 29th ACM International Conference on Multimedia (MM '21), October 20-24, 2021, Virtual Event, China. ACM, New York, NY, USA, 5 pages. https://doi.org/10.1145/3474085.3481028

\section{INTRODUCTION}

Medical imaging has transformed modern medicine, allowing clinicians to noninvasively examine and diagnose patients relatively quickly and easily. Cancer diagnosis via medical imaging is critically important for public health, but it is still far from perfect. For example, in breast cancer diagnosis with mammography, false negative and false positive rates have been reported $6 \%$ to $46 \%$ and approximately $10 \%$, respectively [20]. These errors are mostly due to misperceptions and misinterpretations of $\mathrm{x}$-ray images from radiologists [3,11]. Whereas some sources of errors have been fully identified and characterized (subsequent search misses [5]; low prevalence [13], etc.), errors in cancer image interpretation are still largely without explanation [3]. Given the importance of this issue, a great deal of research has been carried out in the last decades to understand how to identify and characterize the source of these mistakes in order to mitigate them as much as possible.

While interpreting mammograms, radiologists are typically asked to detect any present tumors and classify them as well as record their size, location, and type. Typically, radiologists will examine dozens to hundreds of mammograms one after the other in a short time period. A major underlying assumption is that radiologists' perceptions and decisions on a current mammogram are completely independent of previous perceptual history. However, recent theoretical and empirical research from our lab and others raises the possibility that this is not true. Our visual system is characterized by visual serial dependency (VSD), a type of sequential effect in which what was previously seen influences (captures) what is seen and reported at this moment (Figure 1; [15]).

The visual serial dependence benefits us because the world we live and the scene we experience are usually highly structured, autocorrelated, and stable. However, this situation is not always true. For visual search tasks in mammography, stimuli are not autocorrelated either. Thus, when doing tasks such as tumor localization and classification, serial dependence could introduce a bias in perceptual judgements which would bring in a reduction in sensitivity and increases in errors. The negative impacts of serial dependence in search tasks would be especially prominent in cases where there is low signal, high noise, high uncertainty, or where fine discriminations are required [4, 8, 9, 15, 29, 31]. These are exactly the challenging situations that radiologists routinely face when searching through scans. 
Our pilot data via naive artificial stimuli has shown that VSD has a disruptive effect in radiologic searches that impairs accurate detection and recognition of tumors or other structures [29]. However, this pilot data utilized artificially morphed tumors and simple noisy backgrounds as stimuli which have been noted by both naïve observers and expert radiologists to be less authentic. Recently, Generative Adversarial Networks have been well developed to generate authentic images for certain categories, such as faces, cars, and landscapes $[22,23,34]$. It is intuitive to apply GANs on medical images to generate authentic stimuli for our experiments.

In this project, we will leverage the synthesizing ability of GAN to generate authentic medical images for VSD psychophysical experiments and understand the impact of VSD in visual search tasks for medical image. Moreover, once identifying that VSD has a disruptive effect for medical visual search tasks, we will develop a strategy to mitigate the influence from VSD in clinic settings.

\section{RELATED WORKS}

\subsection{Visual Serial Dependence}

Visual Serial Dependence (VSD) is a common sequential effect of our visual system and it can manifest in several domains, such as perception [9, 15, 31], decision making [1, 14], and memory [25], and they occur with a variety of features and objects, including orientation (Figure 1; [15]), position [4, 31], faces [27, 28, 36, 37], attractiveness [26, 37, 39], ambiguous objects [38], ensemble coding of orientation [30], and numerosity [9, 10]. VSD is characterized by three main kinds of tuning. First, feature tuning: VSD occurs only between similar features [15, 16, 30, 31], and not between dissimilar ones (Figure 1B; [16]). Second, temporal tuning: VSD gradually decays over time (Figure 1C; $[15,31,38]$ ). Third, spatial tuning: VSD occurs only within a limited spatial region and it is strongest when previous and current objects are presented at the same location $[4,15,31]$. In addition, attention is a necessary component for VSD [15].

\subsection{Generative Adversarial Networks}

Generative Adversarial Networks are special Convolutional Neural Networks, which consist of two networks, the generator $(G)$ and the discriminator(D). These two networks are trained iteratively in an adversarial way where the generator $(G)$ generates fake but authentic images to fool the discriminator and the discriminator(D) discriminates the real and fake images [17]. Using this promising computational model, high-quality images with various categories can be generated, such as faces, cars, and landscapes [22, 23, 34]. However, the initial GAN model [17] cannot generate sharp and recognizable images, and the training process is unstable. Later work improved the performance of GAN in different ways. Some papers focus on model architectures [7, 32, 33]. Others focus on improving the loss metrics and training strategies $[2,6,18]$. With these efforts, GAN training stability has improved, and GAN can generate low-resolution images with sufficient quality.

Recently, several approaches make high-resolution image generation also possible. PGGAN [22] proposed to train the standard GAN from coarse to fine scale. The parameters for low-resolution block are trained first. Then higher-resolution blocks are added on gradually with the corresponding parameters updated accordingly.

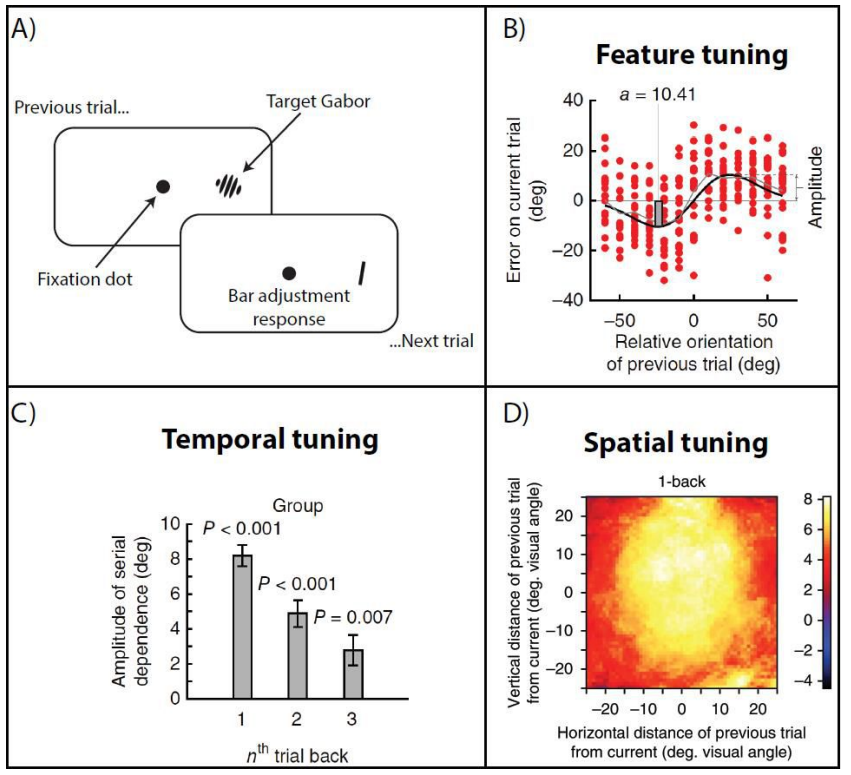

Figure 1: Example of VSD in orientation perception. A) Observers were asked to report the orientation of the Gabor by adjusting a bar when fixating the central dot. B) Error plot as a function of the relative orientation of the previous trial. The Derivative-of-Gaussian fit (DoG) to the data (black line) shows that the perceived orientation was attracted toward the orientation seen on the previous trial. Importantly, VSD is similarity tuned (feature tuning). C) VSD amplitude (gray line in Figure 1B) was significantly positive for up to three trials back, indicating an integration time of 15s (temporal tuning). D) Color coding shows the amplitudes (in degrees) of VSD computed at different locations. Orientation perception was maximally biased toward prior stimuli in the spatial range of $20^{\circ}$ (spatial tuning). Taken from [15].

Based on the same training strategy, StyleGAN [23, 24] proposed to first map the original latent space $\mathcal{Z}$ into the $\mathcal{W}$ space through a non-linear mapping network. Then it is merged into the synthesis network via adaptive instance normalization (AdaIN) at each convolutional block $[12,19]$. This improves StyleGAN representations of scenes and details and allows it to produce authentic high-resolution images. In this project, we adopt StyleGAN as our backbone for medical images generation. Moreover, a controllable approach is also utilized to manipulate the attributes of the generated images.

\section{APPROACH}

In this section, we will illustrate how to generate authentic medical stimuli for visual serial dependence experiments, how to identify visual serial dependence in medical visual search tasks, and how to alleviate its impact in clinic settings.

\subsection{Medical Stimuli Generation}

For medical image synthesizing, we utilize StyleGAN [23]. Additionally, we add an encoder to the network in order to manipulate 
the property of the generated images and importantly to help the calculation of similarity between any pair of images.

While training, the GAN part is first trained progressively [23] via adversarial loss $L_{\text {Adversarial }}$. The training process can be formulated as

$$
\min _{G} \max _{D} E_{x \sim p_{\text {data }}(x)}[\log D(x)]+E_{z \sim q(z)}[\log (1-D(G(z)))]
$$

where $p_{\text {data }}(x)$ and $q(z)$ indicate the real data distribution and the latent space distribution respectively, $x$ is the sampled real image, $z$ is the sampled latent code.

Then, we train the encoder part. After training the GAN part, the generator $(G)$ is fixed. While training the encoder network, we adopt the idea from In-domain GAN inversion [41]. In particular, the encoded vector is passed into the generator $(G)$ again and the regularization is on the reconstructed image. The $\mathrm{L} 2$ reconstruction loss $L_{\text {Reconstruction }}$ and the perceptual loss [21] $L_{\text {Perceptual }}$ are utilized for the regularization. Additionally, adversarial loss $L_{\text {Adversarial }}$ is also utilized to guarantee that the reconstructed image looks authentic. The whole process can be summarized as follows

$$
\begin{aligned}
& \min _{E}\|x-G(E(x))\|_{2}+\lambda_{1}\|F(x)-F(G(E(x)))\|_{2} \\
&-\lambda_{2} E_{x \sim p_{\text {data }}(x)}[\log D(G(E(x)))] \\
& \min _{D} E_{x \sim p_{\text {data }}(x)[\log D(G(E(x)))]}-E_{x \sim p_{\text {data }}(x)}[\log D(x)] \\
&+\frac{\gamma}{2} E_{x \sim p_{\text {data }}(x)}\left[\left\|\nabla_{x} D(x)\right\|_{2}^{2}\right]
\end{aligned}
$$

where $p_{\text {data }}(x)$ indicates the real data distribution, $x$ is the real image, E represents the encoder, F represents the VGG feature extraction [35], and $\lambda_{1}, \lambda_{2}$ and $\gamma$ are weights for the perceptual loss, the adversarial loss, and the gradient penalty [18].

Since the inverse mapping via the encoder(E) will not always be perfect, in order to get the optimal inverse latent code, we apply another optimization on the latent code. This optimization will update the latent code based on the reconstruction loss and the perceptual loss within the neighborhood of the original encoded vector (the encoder regularization). The optimization process can be described as below

$$
\begin{aligned}
z^{i n v}=\min _{z}\|x-G(E(x))\|_{2} & +\lambda_{3}\|F(x)-F(G(z))\|_{2} \\
& +\lambda_{4}\|z-E(G(z))\|_{2}
\end{aligned}
$$

where $z^{i n v}$ is the optimized inverse code, $\lambda_{3}$ and $\lambda_{4}$ are weights for the perceptual loss, and the code reconstruction loss (i.e., the encoder regularization). This optimization metric can be computed using the whole image region (for image reconstruction) or the region of interest (for image manipulation).

After training the whole network, we can utilize the GAN part to synthesize images authentic to real images. First, the latent code $z$ will be sampled from the latent space. Then, the generated image $x=G(z)$ is produced by the generator.

\subsection{Visual Serial Dependence Identification}

In order to identify the existence of visual serial dependence in medical visual search tasks, we first need to find the sequential effect in clinic settings and then test whether these sequential effects are due to VSD, specifically, as recently identified in the literature $[9,15,36,37]$. If so, then the sequential effects in clinical settings should follow the same tuning functions that VSD exhibits more broadly in other previously documented contexts: temporal tuning (Figure 1C; [15, 31, 38]), spatial tuning (Figure 1D; [4, 15, 31]), and featural tuning (Figure 1B; [16]). Moreover, once we know when and how sequential effects are modulated in a clinical task (based on tuning functions), we can implement corrections in Section 3.3 to improve accuracy in diagnostic procedures. To that end, in this section we will investigate the (1) featural, (2) temporal and (3) spatial tuning properties of sequential effects in tumor search order to identify how each tuning property specifically impacts visual search in this clinical task.

Feature tuning Feature tuning means that, the more similar sequential images are, the more serial dependence there is in the perceptual judgments of those objects. On each trial a randomly selected mammogram will be presented. Observers will be asked to adjust a random morph to match the morph they just saw. We will then plot adjustment errors on each trial as a function of relative morph difference and will fit a derivative-of-vonMises function to the data to estimate the strength of VSD (Figure 1B; Previous researches utilize the derivative-of-Gaussian function but derivativeof-vonMises function is more suitable for the cyclic morph pattern).

Temporal tuning Previous results found that VSD is temporally tuned such that feature/object perception is biased based on what was previously presented up to $10-15$ seconds ago [15, 30, 31, 39]. To test this, on each trial a randomly selected mammogram will be presented. Radiologists will be asked to classify the morph as most similar to category. In between trials, we will insert a random delay of $0,8,16$ or 24 seconds.

Spatial tuning The experiment procedure will be the same as for feature tuning and temporal tuning. In addition, the tumor of a mammogram will be presented at a random location.

\subsection{VSD Impact Alleviation}

Once we know when and how sequential effects are modulated in clinical tasks, we will be able to mitigate the impact of VSD with stimulus and procedural manipulations. Here, we propose three different corrections, based on the featural [15, 16, 30, 31], temporal $[15,31,38]$ and spatial $[4,15,31]$ tuning of VSD outlined in Section 3.2. As a result of these manipulations, we will be able to develop tasks for which accuracy in diagnostic procedures will improve.

These corrections can be used to improve any medical visual search task performance. For simplicity, we will outline the classification task as an example of our approach to identify the optimal corrective manipulations. On each trial, observers will classify a randomly selected mammogram as most similar to one of the three categories. As described below, specific task and stimulus modifications will be added to each trial to harness the feature, temporal, and spatial tuning in a beneficial way. 
Feature tuning Previous results have shown that VSD is tuned for feature similarity, and the effect is stronger for more similar images. Thus, we can shuffle the order of a batch of mammograms which would be diagnosed by radiologists. Crucially, this shuffle operation will depend on the mutual similarity among each mammogram in the batch such that the similarity between neighbor mammograms will be minimized. The similarity will be computed via perceptual loss [21] via the encoder of our GAN model.

Temporal tuning To mitigate the effect from temporal tuning mechanism, we will perform an experiment in which we will insert a mask of dynamic noise between each trial, with a temporal delay of $0,8,16$ or 24 seconds and try to find the optimal time delay.

Spatial tuning In this experiment, we will harness spatial tuning to reduce VSD and improve tumor classification sensitivity. Observers will view two computer monitors, rather than one. Each monitor will be separated by at least $50 \mathrm{deg}$ visual angle, to reduce VSD [15].

\section{RESULTS AND WORK IN PROGRESS}

\subsection{GAN Generated Results}

For different medical image modalities, we train the whole network separately using corresponding datasets. After the GAN part has been trained, we randomly sample latent codes $z$ and pass them to the generator. The generated results for Mammogram and CT are shown in Fig 2.

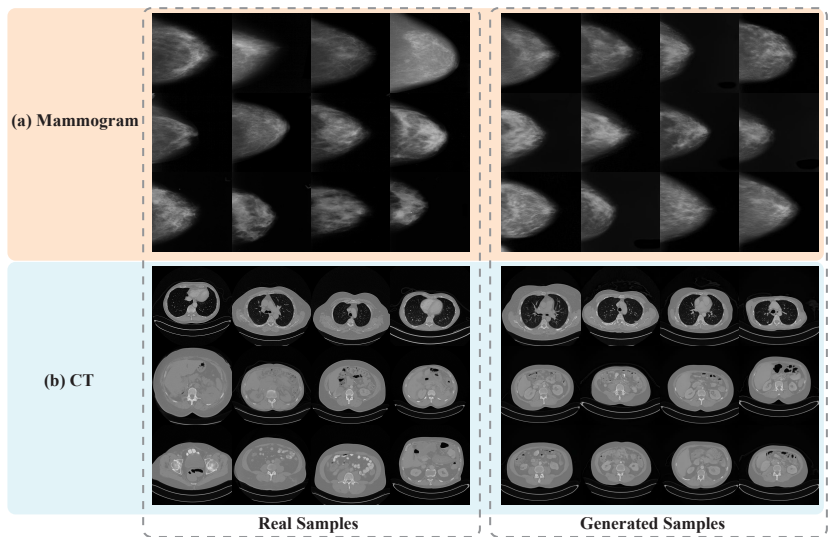

Figure 2: The generated results for mammogram and CT. Comparing the real samples to the generated samples, it is clear that the generator has learned how to imitate tissue texture, tissue distribution and tissue shapes. Thus, it appears to generate authentic images (see below for psychophysical results confirming this).

\subsection{Human Evaluations}

To verify the authenticity of the generated images for different medical image modalities, we conducted an online psychophysical experiment, recruiting both untrained participants (i.e. no knowledge of medical imaging) and experts (e.g. radiologists or practicing clinicians who routinely read radiographs). The task was to rate each image from 0 (fake/generated image) to 10 (real image) in the data pool consisting of equal amount of fake and real images. To ensure that participants did not randomly guess (or lapse), a small number of repetitive trials were also included in the online survey to establish a baseline test-retest reliability estimate.

The results for mammogram and CT images in terms of the Receiver Operating Characteristic (ROC) curves are shown in Fig 3. For both untrained participants and radiologists, and for both mammogram and CT images, their performance curves are near the diagonal (i.e. the chance level performance region), indicating that the generated medical images appeared authentic. From the small number of repeated trials, the average test-retest similarity was 0.65 , indicating highly consistent responses. The similarity is computed using Sokal-Michene metric [40].
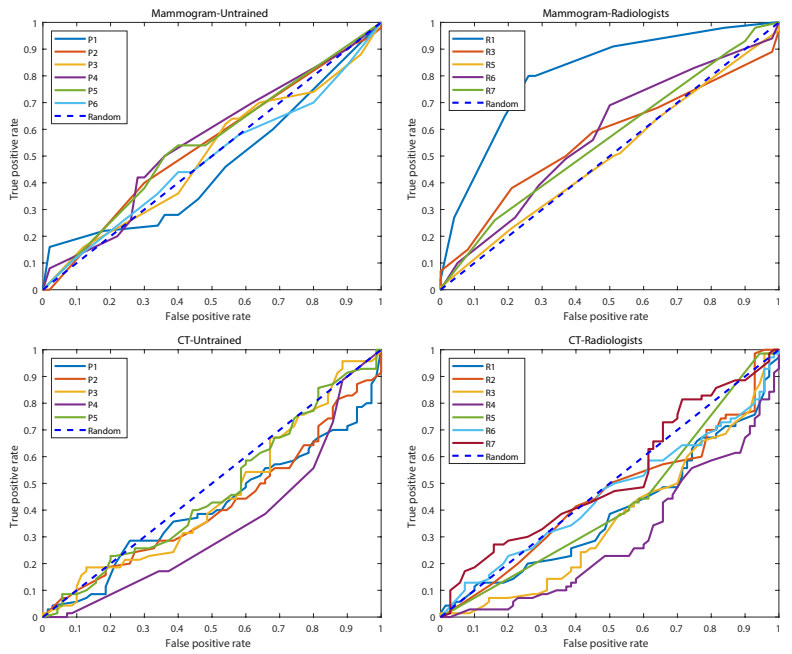

Figure 3: Human evaluation results. Participant performance is shown in the Receiver Operating Characteristic (ROC) curves. It is clear that their performance is near chance level (curves near the diagonal region), indicating that the generated medical images are authentic.

\subsection{Ongoing work}

The generated medical images have been tested to be authentic. Currently, we are recruiting medical image experts as well as untrained observers to conduct the experiments stated in Section 3.2 and Section 3.3. The work includes designing psychophysical experiments and analyze the responses from both kinds of participants.

\section{DISCUSSION}

All the psychophysical experiments are anonymous and voluntary. For the training data of our network, we utilized public datasets where the identity information of all medical images have been removed. The pre-trained model of our project will be provided in the end to ensure reproducibility of the work.

\section{ACKNOWLEDGMENTS}

This work has been supported by National Institutes of Health (NIH) under grant R01CA236793. 


\section{REFERENCES}

[1] Arman Abrahamyan, Laura Luz Silva, Steven C Dakin, Matteo Carandini, and Justin L Gardner. 2016. Adaptable history biases in human perceptual decisions. Proceedings of the National Academy of Sciences 113, 25 (2016), E3548-E3557.

[2] Martin Arjovsky, Soumith Chintala, and Léon Bottou. 2017. Wasserstein generative adversarial networks. In International conference on machine learning. PMLR, 214-223.

[3] Leonard Berlin. 2007. Accuracy of diagnostic procedures: has it improved over the past five decades? American fournal of Roentgenology 188, 5 (2007), 1173-1178.

[4] Daniel P Bliss, Jerome J Sun, and Mark D'Esposito. 2017. Serial dependence is absent at the time of perception but increases in visual working memory. Scientific reports 7, 1 (2017), 1-13.

[5] B Boyer, L Hauret, R Bellaiche, C Gräf, B Bourcier, and G Fichet. 2004. Retrospectively detectable carcinomas: review of the literature. Fournal de radiologie 85,12 Pt 2 (2004), 2071-2078.

[6] Andrew Brock, Jeff Donahue, and Karen Simonyan. 2018. Large scale GAN training for high fidelity natural image synthesis. arXiv preprint arXiv:1809.11096 (2018).

[7] Xi Chen, Yan Duan, Rein Houthooft, John Schulman, Ilya Sutskever, and Pieter Abbeel. 2016. InfoGAN: interpretable representation learning by information maximizing generative adversarial nets. In Proceedings of the 30th International Conference on Neural Information Processing Systems. 2180-2188.

[8] Guido Marco Cicchini, Giovanni Anobile, and David C Burr. 2014. Compressive mapping of number to space reflects dynamic encoding mechanisms, not static logarithmic transform. Proceedings of the National Academy of Sciences 111, 21 (2014), 7867-7872.

[9] Guido Marco Cicchini, Kyriaki Mikellidou, and David Burr. 2017. Serial dependencies act directly on perception. Fournal of Vision 17, 14 (2017), 6-6.

[10] Jennifer E Corbett, Jason Fischer, and David Whitney. 2011. Facilitating stable representations: Serial dependence in vision. PLoS One 6, 1 (2011).

[11] Pat Croskerry. 2003. The importance of cognitive errors in diagnosis and strategies to minimize them. Academic medicine 78, 8 (2003), 775-780.

[12] Vincent Dumoulin, Jonathon Shlens, and Manjunath Kudlur. 2016. A learned representation for artistic style. arXiv preprint arXiv:1610.07629 (2016)

[13] Karla K Evans, Robyn L Birdwell, and Jeremy M Wolfe. 2013. If you don't find it often, you often don't find it: why some cancers are missed in breast cancer screening. PloS one 8, 5 (2013).

[14] Samuel W Fernberger. 1920. Interdependence of judgments within the series for the method of constant stimuli. Journal of Experimental Psychology 3, 2 (1920), 126 .

[15] Jason Fischer and David Whitney. 2014. Serial dependence in visual perception Nature neuroscience 17, 5 (2014), 738-743.

[16] Matthias Fritsche, Pim Mostert, and Floris P de Lange. 2017. Opposite effects of recent history on perception and decision. Current Biology 27, 4 (2017), 590-595.

[17] Ian J Goodfellow, Jean Pouget-Abadie, Mehdi Mirza, Bing Xu, David Warde-Farley, Sherjil Ozair, Aaron Courville, and Yoshua Bengio. 2014. Generative adversarial networks. arXiv preprint arXiv:1406.2661 (2014).

[18] Ishaan Gulrajani, Faruk Ahmed, Martin Arjovsky, Vincent Dumoulin, and Aaron Courville. 2017. Improved training of wasserstein gans. arXiv preprint arXiv:1704.00028 (2017)

[19] Xun Huang and Serge Belongie. 2017. Arbitrary style transfer in real-time with adaptive instance normalization. In Proceedings of the IEEE International Conference on Computer Vision. 1501-1510.

[20] National Cancer Institution. 2020. Breast Cancer Screening, Health Professional Version. https://www.cancer.gov/types/breast/hp/breast-screening-pdq

[21] Justin Johnson, Alexandre Alahi, and Li Fei-Fei. 2016. Perceptual losses for realtime style transfer and super-resolution. In European conference on computer vision. Springer, 694-711.

[22] Tero Karras, Timo Aila, Samuli Laine, and Jaakko Lehtinen. 2017. Progressive growing of gans for improved quality, stability, and variation. arXiv preprint arXiv:1710.10196 (2017).

[23] Tero Karras, Samuli Laine, and Timo Aila. 2019. A style-based generator architecture for generative adversarial networks. In Proceedings of the IEEE/CVF Conference on Computer Vision and Pattern Recognition. 4401-4410.

[24] Tero Karras, Samuli Laine, Miika Aittala, Janne Hellsten, Jaakko Lehtinen, and Timo Aila. 2020. Analyzing and improving the image quality of stylegan. In Proceedings of the IEEE/CVF Conference on Computer Vision and Pattern Recognition. 8110-8119.

[25] Anastasia Kiyonaga, Jason M Scimeca, Daniel P Bliss, and David Whitney. 2017. Serial dependence across perception, attention, and memory. Trends in Cognitive Sciences 21, 7 (2017), 493-497.

[26] Aki Kondo, Kohske Takahashi, and Katsumi Watanabe. 2012. Sequential effects in face-attractiveness judgment. Perception 41, 1 (2012), 43-49.

[27] Alina Liberman, Jason Fischer, and David Whitney. 2014. Serial dependence in the perception of faces. Current biology 24, 21 (2014), 2569-2574.

[28] Alina Liberman, Mauro Manassi, and David Whitney. 2018. Serial dependence promotes the stability of perceived emotional expression depending on face similarity. Attention, Perception, \& Psychophysics 80, 6 (2018), 1461-1473.

[29] Mauro Manassi, Árni Kristjánsson, and David Whitney. 2017. Serial dependence determines object classification in visual search. Fournal of Vision 17, 10 (2017), 221-221.

[30] Mauro Manassi, Alina Liberman, Wesley Chaney, and David Whitney. 2017. The perceived stability of scenes: serial dependence in ensemble representations. Scientific reports 7, 1 (2017), 1-9.

[31] Mauro Manassi, Alina Liberman, Anna Kosovicheva, Kathy Zhang, and David Whitney. 2018. Serial dependence in position occurs at the time of perception. Psychonomic Bulletin \& Review 25, 6 (2018), 2245-2253.

[32] Mehdi Mirza and Simon Osindero. 2014. Conditional generative adversarial nets. arXiv preprint arXiv:1411.1784 (2014)

[33] Augustus Odena, Christopher Olah, and Jonathon Shlens. 2017. Conditional image synthesis with auxiliary classifier gans. In International conference on machine learning. PMLR, 2642-2651.

[34] Taesung Park, Ming-Yu Liu, Ting-Chun Wang, and Jun-Yan Zhu. 2019. Semantic image synthesis with spatially-adaptive normalization. In Proceedings of the IEEE/CVF Conference on Computer Vision and Pattern Recognition. 2337-2346.

[35] Karen Simonyan and Andrew Zisserman. 2014. Very deep convolutional networks for large-scale image recognition. arXiv preprint arXiv:1409.1556 (2014).

[36] Jessica Taubert, David Alais, and David Burr. 2016. Different coding strategies for the perception of stable and changeable facial attributes. Scientific reports 6 (2016), 32239.

[37] Jessica Taubert, Erik Van der Burg, and David Alais. 2016. Love at second sight: Sequential dependence of facial attractiveness in an on-line dating paradigm. Scientific reports 6, 1 (2016), 1-5.

[38] Mark Wexler, Marianne Duyck, and Pascal Mamassian. 2015. Persistent states in vision break universality and time invariance. Proceedings of the National Academy of Sciences 112, 48 (2015), 14990-14995.

[39] Ye Xia, Allison Yamanashi Leib, and David Whitney. 2016. Serial dependence in the perception of attractiveness. Fournal of vision 16, 15 (2016), 28-28.

[40] Bin Zhang and Sargur N Srihari. 2003. Properties of binary vector dissimilarity measures. In Proc. FCIS Int'l Conf. Computer Vision, Pattern Recognition, and Image Processing, Vol. 1.

[41] Jiapeng Zhu, Yujun Shen, Deli Zhao, and Bolei Zhou. 2020. In-domain gan inversion for real image editing. In European Conference on Computer Vision. Springer, 592-608. 